\title{
Mise en place du Projet de Gestion et de Soutien dans un Etablissement Public Hospitalier : cas de l'Hôpital du Mali
}

\author{
Oumar Bah \\ Docteur en Sciences de Gestion \\ Relecteur - Enseignant - Chercheur à l'Université Sup' Management, Mali \\ Boubacar Sanogo \\ Docteur en Sciences de Gestion \\ Relecteur - Enseignant - Chercheur à l’Université de Ségou, Mali \\ Toumani Bagayoko \\ Maître de Conférences à la FSEG et à l'USSGB
}

\begin{abstract}
Doi:10.19044/esj.2020.v16n28p213 URL:http://dx.doi.org/10.19044/esj.2020.v16n28p213
Submitted: 29 May 2020

Accepted: 14 August 2020

Published: 31 October 2020

Copyright 2020 Bah et al.

Distributed under Creative Commons CC-BY 4.0

OPEN ACCESS
\end{abstract}

\section{Résumé}

Cet article est une recherche sur la mise en place du Projet de Gestion à l'Hôpital du Mali. Dans le cadre de l'élaboration du Projet d'Etablissement de l'Hôpital du Mali (BAH, 2019), le projet de gestion et de soutien occupe une place très importante et le corps administratif et de gestion constituent le socle de ce projet. De ce fait, l'objectif de cette étude est de mettre en œuvre la politique définie par les autorités de tutelle en matière d'organisation, de gestion administrative, financière et comptable dans les Etablissements Publics Hospitaliers (EPH) du Mali. A cet égard, le projet de gestion et de soutien devra permettre un recueil d'informations nécessaires pour une utilisation rationnelle des ressources financières de l'Hôpital du Mali. Le projet de gestion et de soutien de l'Hôpital du Mali, dans le cadre du Projet d'Etablissement Hospitalier comprend deux commissions en plus de la Direction Générale ; il s'agit du Comité Technique d'Etablissement (CTE) et du Comité Technique d'Hygiène et de Sécurité (CTHS). Le Comité Technique d'Etablissement (CTE) est chargé d'étudier et de donner son avis sur les questions relatives aux conditions de travail. Le Comité Technique d'Hygiène et de Sécurité (CTHS) est chargé d'étudier et de donner des avis sur la protection, l'hygiène, la sécurité des soins, des personnes et des biens au sein de l'Hôpital (Loi n 10-010 AN-RM du 20 mai 2010). Pour l'atteinte des objectifs, nous avons utilisé une approche qualitative. Sur un effectif total de 143 agents, nous avons tiré un échantillon de 58 agents. La méthodologie utilisée dans cette recherche a été : l'administration d'un questionnaire au personnel de gestion et de soutien, le recueil et le traitement des résultats conduisant à la réalisation du plan d'actions qui regroupe l'ensemble des besoins (en formation, en matériels et équipements, et en 
aménagement). La finalité du projet de gestion et de soutien de l'Hôpital du Mali est l'amélioration des conditions de gestion et de soutien à l'Hôpital du Mali (ANEH, octobre 2010).

Mots clés: Projet de Gestion et de Soutien (PGS), Comité Technique d'Etablissement (CTE), Comité Technique d'Hygiène et de Sécurité (CTHS). 


\title{
Implementation of The Management and Support Project in A Public Hospital Establishment: Case of The Mali Hospital
}

\author{
Oumar Bah \\ Docteur en Sciences de Gestion \\ Relecteur - Enseignant - Chercheur à l'Université Sup' Management, Mali \\ Boubacar Sanogo \\ Docteur en Sciences de Gestion \\ Relecteur - Enseignant - Chercheur à l'Université de Ségou, Mali \\ Toumani Bagayoko \\ Maître de Conférences à la FSEG et à l'USSGB
}

\begin{abstract}
This article is a research on the implementation of the Management Project at the Mali Hospital. Within the framework of the development of the Mali Hospital Establishment Project (BAH, 2019), the management and support project occupies a very important place and the administrative and management body is the foundation of this project. Therefore, the objective of this study is to implement the policy defined by the supervisory authorities in terms of organization, administrative, financial and accounting management in Mali's Public Hospital Establishments (PHE). In this respect, the management and support project should enable the collection of information necessary for the rational use of the financial resources of the Mali Hospital. The Mali Hospital Management and Support Project, within the framework of the Hospital Establishment Project, includes two commissions in addition to the General Management; these are the Technical Committee for Establishment (TCE) and the Technical Committee for Health and Safety (TCHS). The Technical Committee for Establishment (TCE) is responsible for studying and giving its opinion on matters relating to working conditions. The Technical Committee on Health and Safety (TCHS) is responsible for studying and giving opinions on the protection, hygiene, safety of care, persons and property within the Hospital. To achieve the objectives, we used a qualitative approach. From a total staff of 143 agents, we selected a sample of 58 agents. The methodology used in this research was: the administration of a questionnaire to management and support staff, the collection and processing of the results leading to the implementation of an action plan that brings together all the needs (in training, materials and equipment, and development). The aim of the Mali Hospital Management
\end{abstract}


and Support Project is to improve management and support conditions at the Mali Hospital.

Keywords: Management and Support Project (MSP), Technical Establishment Committee (TEC), Technical Committee on Health and Safety (TCHS).

\section{Introduction}

L'Hôpital du Mali est un Etablissement Public Hospitalier (EPH) dans la nomenclature des hôpitaux au Mali ayant pour mission : d'assurer le diagnostic, le traitement des malades et des blessés, de prendre en charge les urgences et les cas référés, d'assurer la formation initiale et la formation continue des professionnels de la santé, et de conduire des travaux de recherche dans le domaine médical.

Le Projet de Gestion et de Soutien (PGS) de l'Hôpital du Mali, dans le cadre du Projet d'Etablissement Hospitalier (PEH) est sous l'apanage de deux commissions, à savoir : le Comité Technique d'Etablissement (CTE) et le Comité Technique d'Hygiène et de Sécurité (CTHS).

Le décret $\mathrm{n}^{\circ} 10-316 / \mathrm{P}-\mathrm{RM}$ du 03 juin 2010 fixant l'organisation et les modalités de fonctionnement de l'Hôpital du Mali stipule dans sa section 3 (Articles 23 à 27) la mise en place du Comité Technique d'Etablissement (CTE). Le Comité Technique d'Etablissement (CTE) est chargé d'étudier et de donner son avis sur les questions relatives aux conditions de travail. Le Comité Technique d' Etablissement comprend entre autre : le Directeur Général de l'hôpital (DG) et les représentants élus par collège dans chacun des six collèges suivants : deux représentants du collège des cadres A médicaux ; un représentant du collège des cadres A non médicaux ; deux représentants du collège des cadres $\mathrm{B}$ paramédicaux ; un représentant du collège des cadres $\mathrm{B}$ non médicaux ; deux représentants du collège autres personnels de soins ; un représentant du collège «autres personnels ».

Le décret $\mathrm{n}^{\circ} 10-316 / \mathrm{P}-\mathrm{RM}$ du 03 juin 2010 fixant l'organisation et les modalités de fonctionnement de l'Hôpital du Mali stipule dans sa section 4 (Articles 28 à 33) la mise en place du Comité Technique d'Hygiène et de Sécurité (CTHS) de l'Hôpital du Mali. Le Comité Technique d'Hygiène et de Sécurité (CTHS) est chargé d'étudier et de donner des avis sur la protection de l'hygiène, la sécurité des soins et celle des personnes et des biens au sein de l'Hôpital. Le Comité Technique d'Hygiène et de Sécurité (CTHS) comprend deux représentants élus par chacune des catégories suivantes : médecins, pharmaciens, biologistes ; assistants médicaux ; techniciens supérieurs ; auxiliaires de santé ; agents administratifs ; agents de surface ; techniciens d'hygiène et les agents sociaux. 
Objectif général : L'objectif général du projet de gestion et de soutien de l'hôpital du Mali se définit comme suit : renforcer les capacités de gestion et de soutien de l'hôpital à travers la mise en place du Projet de gestion et de soutien.

Objectifs spécifiques : Les objectifs spécifiques du projet de gestion et de soutien de l'hôpital du Mali se résument en quatre (4) points comme suit :

- OS1 - Renforcer le personnel de gestion et de soutien;

- OS2 - Renforcer les capacités du personnel de gestion et de soutien;

- OS3 - Renforcer la maintenance, l'acquisition des matériels et équipements de gestion et de soutient ;

- OS4 - Renforcer les moyens logistiques dans les services.

Problème : Insuffisance des conditions de gestion et de soutien de l'hôpital du Mali est le développement des capacités et des moyens de gestion.

Méthodologie : la méthodologie utilisée dans la réalisation du Projet de Gestion et de Soutien (PGS) de l'Hôpital du Mali peut se résumer en trois point, à savoir: l'administration du questionnaire suivi d'une discussion personnalisée avec les chefs de service administratifs. Le recueil et le traitement des données en vue de la réalisation du plan d'actions du projet du Projet de Gestion et de Soutien (PGS) de l'Hôpital du Mali.

Résultat attendu : Le résultat attendu du projet de gestion et de soutien de l'hôpital du Mali est le développement des capacités et des moyens de gestion.

Questions de recherche : la présente étude se propose de répondre aux questions suivantes :

Le Projet de Gestion et de Soutien (PGS) contribuera il à l'amélioration de la gestion hospitalière et des activités de soutien de l'Hôpital du Mali ? Les renforcements des capacités des agents de la gestion hospitalière et des activités de soutien (formations, matériels et équipements, infrastructures) auront-ils des répercutions sur le management de l'Hôpital du Mali ?

\section{Les hypothèses de recherche :}

Hypothèse 1: l'amélioration des aspects de gestion et de soutien permettra à l'Hôpital du Mali de rehausser la qualité du management hospitalier.

Hypothèse 2: Les renforcements des capacités des agents de la gestion hospitalière et des activités de soutien de l'Hôpital du Mali apporteront des améliorations dans le management. 


\section{1- La Méthodologie d'élaboration du Projet de Gestion et de Soutien (PGS)}

1.1 - Site de l'étude : Le site de cette étude est l'Hôpital du Mali, un Etablissement Public Hospitalier (EPH) situé au quartier Missabougou, dans la commune VI du district de Bamako, Mali. Bâti à la sortie Sud du pont de l'amitié Mali-Chine ( $3^{\mathrm{e}}$ pont de Bamako), à quelques mètres des berges du fleuve Niger, à la sortie sud-est de la ville de Bamako, ce véritable joyau architectural a été construit et équipé en grande partie par le partenaire chinois. L'Hôpital du Mali a une capacité actuelle de cent cinquante (150) lits dont cent trente un (131) lits d'hospitalisation. Cette capacité doit progressivement atteindre quatre cent (400) lits grâce à la disponibilité d'une superficie de vingt (20) hectares.

\section{Figure 1 : Carte de localisation par satellite de l'Hôpital du Mali}

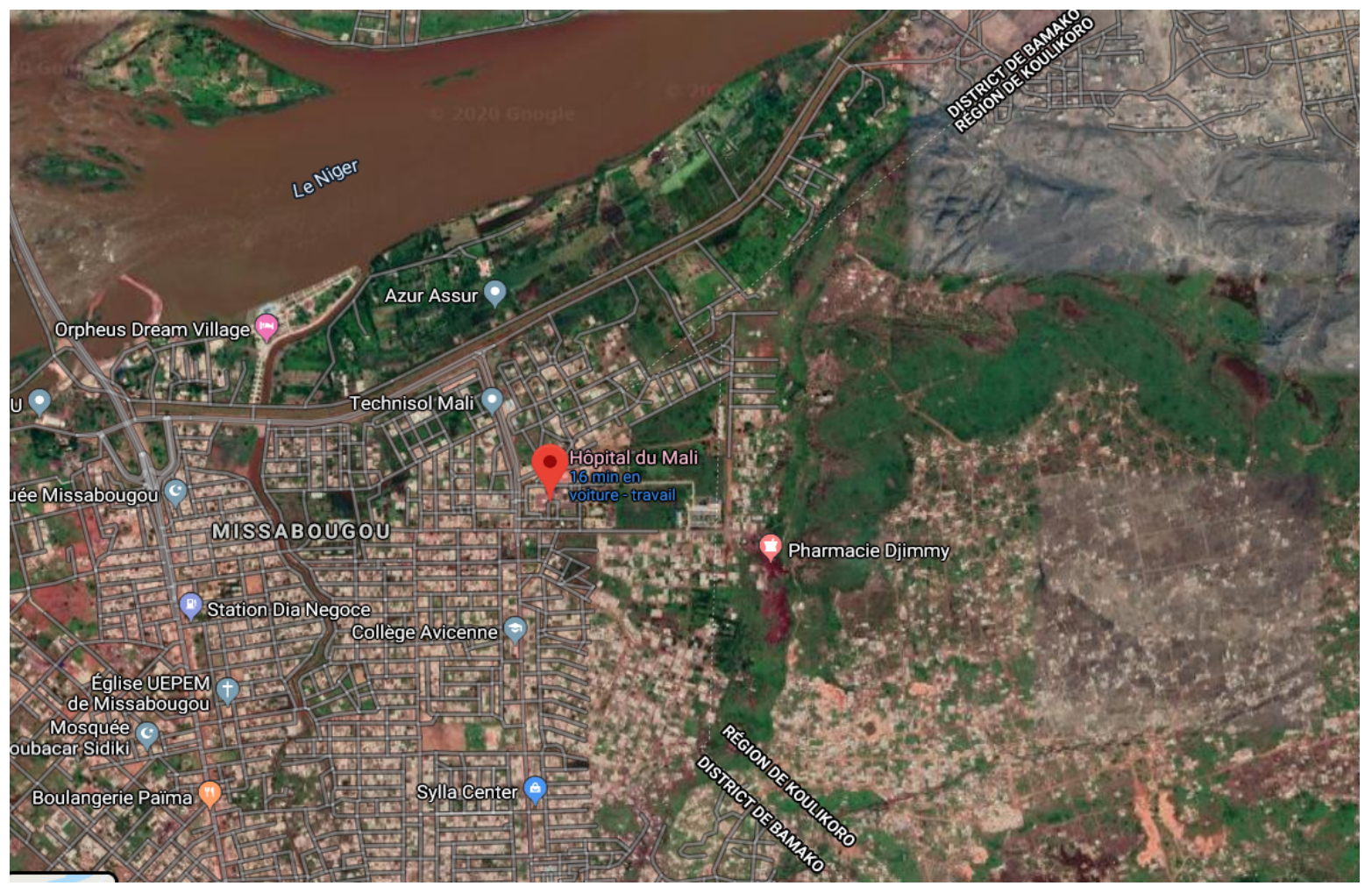

Source :

https://www.google.com/maps/place/H\%C3\%B4pital+du+Mali/@12.633936

6,7.9113042,3059m/data $=! 3 \mathrm{~m} 1 ! 1 \mathrm{e} 3 ! 4 \mathrm{~m} 5 ! 3 \mathrm{~m} 4 ! 1 \mathrm{~s} 0 \times 0: 0 \times 4 \mathrm{c} 71 \mathrm{a} 4 \mathrm{a} 7144 \mathrm{ac} 0 \mathrm{e} 7 !$ $\underline{8 m 2 ! 3 \mathrm{~d} 12.6339366 ! 4 \mathrm{~d}-7.9113042}$ 
1.2 - Collecte et analyse des données : Le projet de Gestion et de Soutien a pour but l'identification des problèmes de gestion et de soutien et la recherche des solutions adéquates. Il est réalisé par dans l'optique d'une optimisation du couple «coût/qualité » des soins qui constitue l'un des piliers fondamentaux des Etablissements Publics Hospitaliers (EPH) au Mali. Un débat a été initié au sein de l'Hôpital du Mali, amenant chaque agent de gestion et de soutien à s'interroger sur les missions et les obstacles qui, selon lui, empêchent ce dernier de les remplir correctement. Le choix d'un mode d'organisation par service nous a permis de commencer à créer l'état d'esprit nécessaire au travail en équipe. Ces réflexions ont été réalisées sous la responsabilité de chaque chef de service, qui devait réunir l'ensemble de son personnel de gestion ou de soutien pour des séances organisées, jusqu'à la remise du rapport final. Au cours de ces réflexions, chaque agent devrait s'exprimer, quelle que soit sa qualification et sa responsabilité au sein du service.

Le programme fut réalisé en trois (3) étapes.

Une première étape fut destinée à l'identification des problèmes qui empêchent l'Hôpital du Mali de réaliser correctement ses missions. La liberté de parole a été totale et tous les sujets ont été évoqués, y compris les problèmes d'ordre personnel, s'ils ont une incidence sur le fonctionnement de l'Hôpital du Mali. Les agents ont été notamment invités à : identifier les problèmes qui limitent ses performances et qui s'opposent à ce qu'il remplisse correctement ses missions; identifier les problèmes qui entravent la qualité des services de gestion et de soutien; identifier les problèmes qui sont à l'origine des dépenses excessives; etc.

Aucun problème identifié n'a été exclu; dans la mesure où il peut constituer un obstacle majeur à la performance ou au développement de l'Hôpital (c'est notamment le cas des problèmes liés aux situations individuelles dans le bon fonctionnement, c'est à dire y compris les problèmes de logement, de déplacement, de gardes d'enfants, de revenus, etc....).

La réalisation de cette première étape a conduit les chefs de service à élaborer la liste de tous les problèmes évoqués, à les classer et à les sélectionner au terme d'un débat avec le personnel de gestion et de soutien du service. Les problèmes retenus ont été listés, discutés et classés par ordre de priorité.

Une deuxième étape a permis à chaque agent de gestion et de soutien de proposer des solutions pour chaque problème identifié. Les solutions proposées furent listées, discutées et regroupées par catégorie. Pour chaque problème identifié, les solutions envisageables furent proposées. Ces solutions ont été souvent ponctuelles et d'ordre général. Elles ont été orientées vers l'action et replacées dans le système que constitue l'hôpital du 
Mali proprement dit d'une part, et de l'Hôpital du Mali dans son environnement d'autre part.

Une troisième étape a permis à chaque agent de gestion et de soutien de faire ses recommandations sur toutes les mesures d'ordre général qui doivent être prises pour permettre à l'Hôpital du Mali d'apporter des solutions aux problèmes posés et de mieux remplir ses missions. Une synthèse de l'analyse interne (analyse SWOT) présentant un classement des problèmes et des solutions retenues pour chacun d'eux fut effectuée.

\section{3 - Modalités d'élaboration du Projet de Gestion et de Soutien (PGS)}

Le Comité Technique d'Etablissement (CTE) est composée des représentants élus par collège dans chacun des six (6) collèges suivants : - Deux (2) représentants du collège des cadres A médicaux ; - Un (1) représentant du collège des cadres A non médicaux ; - Deux (2) représentants du collège des cadres B paramédicaux ; - Un (1) représentant du collège des cadres B non médicaux ; - Deux (2) représentants du collège autres personnels de soins ; - Un (1) représentant du collège « autres personnels ».

Il a pour rôle d'étudier et de donner son avis sur les questions relatives aux conditions de travail.

Le Comité Technique d'Hygiène et de Sécurité (CTHS) comprend deux (2) représentants élus par chacune des catégories suivantes:

- Médecins, pharmaciens, biologistes ;

- Assistants médicaux ;

- Techniciens supérieurs ;

- Auxiliaires de santé ;

- Agents administratifs ;

- Agents de surface ;

- Techniciens d'hygiène ;

- Agents sociaux.

Il est chargé d'étudier et de donner des avis sur la protection de l'hygiène, la sécurité des soins et celle des personnes et des biens au sein de l'Hôpital.

Ces deux comités valident les étapes de la conception du Projet de Gestion et de Soutien (PGS). Les professionnels la composant ont été choisis pour participer aux différents groupes de travail. La Direction Générale a voulu que cet organe joue un rôle important dans l'élaboration mais aussi dans le suivi du Projet de Gestion et de Soutien (PGS) l'Hôpital du Mali. Chaque projet fait l'objet d'une fiche dite «projet » qui précise les différentes actions, le calendrier de mise en œuvre et de son suivi par service technique dûment élaboré par le Directeur Général Adjoint (DGA). L'encadrement administratif (chef de service) est régulièrement informé de 
l'avancée des travaux et il répercute l'information aux membres de son service.

1.4 - L'échantillonnage : Pour atteindre les objectifs de l'étude, nous avons utilisé une approche qualitative. Sur un effectif de 143 agents, nous avons tiré 58 agents. L'utilisation du questionnaire a permis de collecter des données d'analyse. L'échantillonnage à concerner 58 responsables de la structure. Le tableau suivant retrace le processus de l'administration du questionnaire.

\begin{tabular}{|l|l|}
\hline Catégories consultees & Nombre \\
\hline Directeur Général & 01 \\
\hline Directeur Général Adjoint & 01 \\
\hline Sécretariat Général & 02 \\
\hline Agence Comptable & 04 \\
\hline Comptable Matières & 01 \\
\hline Auditeur Interne & 01 \\
\hline Delegué du Contrôle Financier & 01 \\
\hline Ressources Humaines & 01 \\
\hline Surveuillant Général & 01 \\
\hline Service Social & 01 \\
\hline Maintenance & 01 \\
\hline Santé Publique (SIH) & 01 \\
\hline Informatique & 01 \\
\hline Pharmacie hospitalière (CTHS) & 01 \\
\hline Chefs de Services Techniques (Médecins) & 20 \\
\hline Surveillants de services & 20 \\
\hline Total & $\mathbf{5 8}$ \\
\hline
\end{tabular}

Source : Cabinet Auditec Fiduciaire, (année 2014), pour la réalisation du

Projet d'Accueil, de Soins Infirmiers et Obstétricaux (PASIO), Page 23.

Ainsi, il a été administrer au personnel interne de l'hôpital un questionnaire pour faire ressortir avec exactitude les problèmes et les propositions de solution provenant directement des agents de l'Hôpital du Mali. Cet échantillonnage de type qualitative a abouti à la réalisation du plan d'action du projet de gestion et de soutien. Le questionnaire ci haut cité est présenté en annexe. 


\section{2 - Aspects de gestion de l'Hôpital du Mali}

Il faut signaler que la Direction Général coordonne l'ensemble des activités de l'hôpital.

\section{1 - Gestion des bâtiments de l'Hôpital du Mali}

Notons que, l'article 4 du Décret $n^{\circ} 10-681 / \mathrm{PM}$ RM du 30 décembre2010 portant règlementation de la comptabilité matières, stipule que la comptabilité des propriétés immobilières bâties et non bâties (bâtiments à usage de bureau, les logements administratifs civils et militaires, les bâtiments à usage techniques et les terrains nus) est tenue par la conservation du domaine, du cadastre et de la curatelle, en rapport avec les bureaux comptables et le service de l'habitat. De ce fait, il faut des techniciens pour procéder à la mise en place des fiches matricules de propriété immobilière de l'Hôpital du Mali.

\section{2 - Gestion des équipements et matériels}

Selon le décret $\mathrm{n}^{\circ} 10-681 / \mathrm{PM}$ RM du 30 décembre2010 portant règlementation de la comptabilité matières et l'arrêté $n^{\circ} 2011-4795 / \mathrm{MEF}$ SG du 25 novembre 2011 fixant les modalités d'application dudit décret $\mathrm{n}^{\circ} 10$ 681/PM-RM du 30 décembre2010, les équipements (en approvisionnement ou en service) sont gérés par le comptable-matières dont les actes sont supervisés et approuvés par l'ordonnateur-matières. L'Hôpital du Mali, a ouvert ses portes le 07 septembre 2011 avec quelques difficultés avérées en matière d'infrastructures, de mobiliers de bureau, de matériels médicaux et de consommables médicaux. Cette dotation initiale composée de dons de la République Populaire de Chine et de la contribution de l'Etat malien s'avère insuffisante et doit bénéficier d'une attention particulière pour l'acquisition de nouveaux équipements. Le choix des nouveaux équipements se fait à partir des besoins exprimés par les différents services et validés par la Direction Générale en fonction de la disponibilité des fonds. Les fiches d'inventaires existent et sont tenues à jour au niveau de la comptabilitématières de l'hôpital. Une fiche technique existe pour chaque équipement et des fiches détenteurs existent par service.

\section{3 - Gestion des Ressources Humaines}

Les ressources humaines constituent un élément essentiel et stratégique de toute activité de production. La maîtrise de sa gestion est ainsi un facteur de succès pour l'hôpital du Mali.

Le dossier administratif individuel est en cours d'élaboration pour chaque agent de l'hôpital contenant les informations d'indentifications nécessaires (extrait de naissance, acte de mariage, les extraits des enfants, l'arrêté d'intégration, la décision de titularisation, l'attestation de prise de 
service, la décision d'affectation, le diplôme de base, et tout autre diplôme obtenu en cours de carrière). Comme modalités de motivation des agents hospitaliers, l'Hôpital du Mali donne des ristournes chaque trimestre à l'ensemble du personnel. Le tableau ci-dessous retrace les motivations financières reçues par le personnel de l'Hôpital du Mali. Un plan de formation est en cours d'élaboration qui est établi sur la demande des agents et validé par le Directeur Général en fonction de la disponibilité des fonds. Il existe des fiches de descriptions de poste et de tâche (qui ne sont pas encore validées). Une organisation des congés existe et le congé annuel est accordé au personnel après service fait en raison d'un mois de repos pour onze mois de service. Outre les congés annuels, les femmes, à l'occasion de leur accouchement bénéficient d'un congé de maternité de quatorze semaines en raison de six avant et huit après l'accouchement.

Toute absence autre que celle prévue par le code de travail et le statut général de la fonction publique doit faire l'objet d'une autorisation préalable du Directeur Général de l'Hôpital du Mali. Elle fera en outre l'objet d'une compensation ou d'une déduction des congés annuels, lorsque le nombre total des jours d'absence dans l'année est égal ou supérieur à quinze jours.

La garde débute à seize heures et se termine le lendemain à huit heures pour les agents de santé. La garde débute le vendredi à douze heures trente minutes et s'étale sur vingt-quatre heures les samedis, dimanches et jours fériés. Toute modification de la liste de garde doit être notifiée au Surveillant et dans les meilleurs délais. Aucun agent n'a le droit d'abandonner son poste pendant la garde et avant l'arrivée de son remplaçant. Pour un meilleur suivi des malades et des urgences, la passation entre les équipes descendantes et montante est exigée. Dans le cadre du projet d'établissement de l'hôpital du Mali, un organigramme fonctionnel a été proposé, il se présente comme suit: 
ORGANIGRAMME DE L'HOPITAL DU MALI

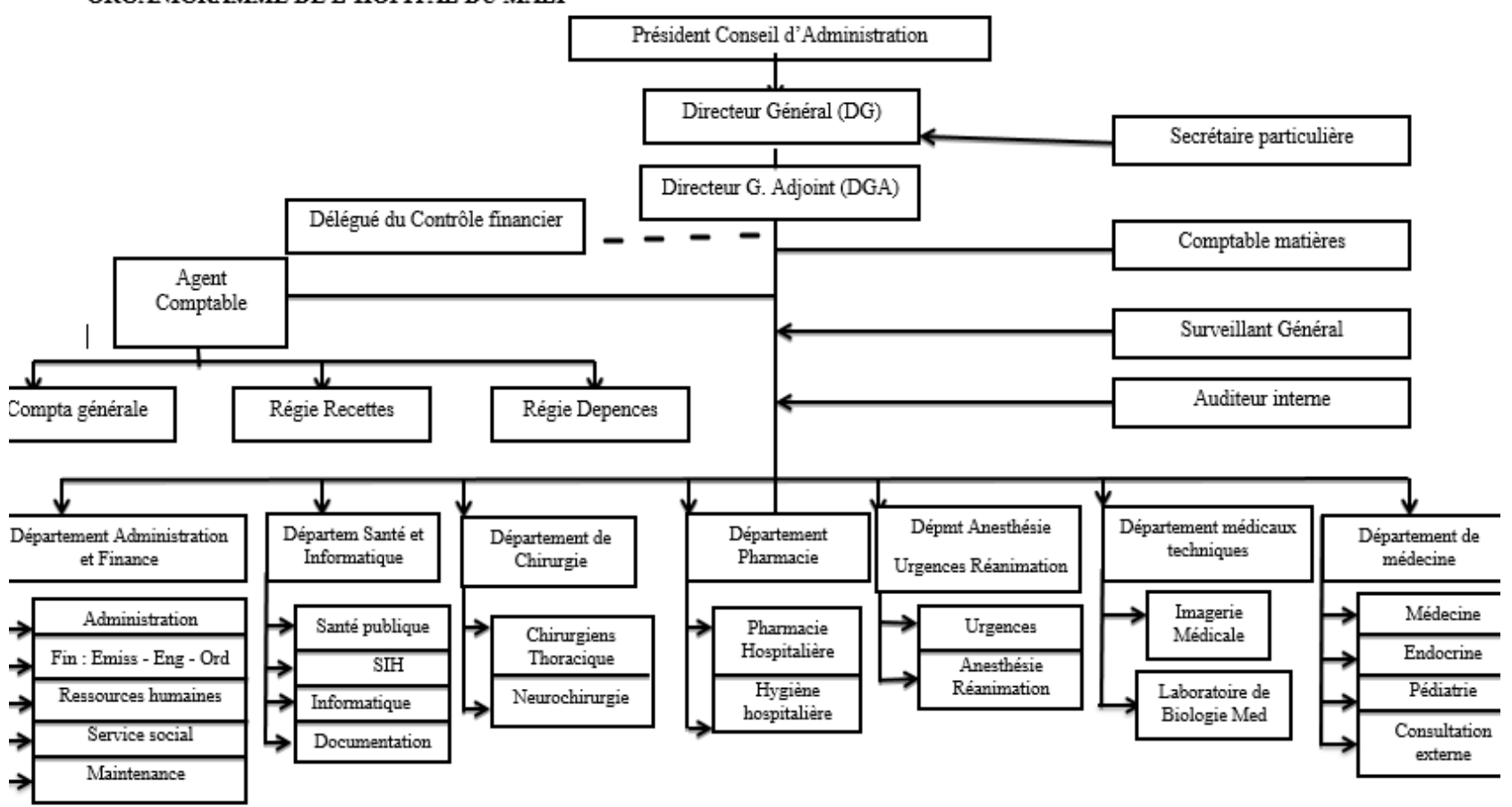

\section{4 - Gestion financière de l'Hôpital du Mali}

L'hôpital du Mali est un organisme doté de la personnalité morale et de l'autonomie financière, son budget se répartit entre les dépenses de personnel, dépenses de matériel et les dépenses d'investissement. En règle générale, toute dépense faite au cours d'un exercice doit être conforme aux prévisions budgétaires dont le suivi est assuré par le Service Administratif et Financier (SAF), l'agent comptable et le contrôleur financier. Le budget retrace les activités en termes de quantité et de masse financière pour un exercice budgétaire allant du $1^{\text {er }}$ janvier au 31 décembre. Des rapports d'activités annuels sont établis chaque année par le Service Administratif et Financier (SAF) et le suivi est assuré par l'agent comptable.

\section{5 - Gestion des paiements des patients}

Les modalités de paiement des prestations au niveau de l'hôpital sont comme suit :

L'usager se présente d'abord à l'accueil - orientation où il est guidé selon son statut.

- Si c'est un patient ordinaire, il est dirigé vers la caisse pour se faire enregistrer et payer le ticket ; 
- Si c'est un patient affilié à l'Assurance Maladie Obligatoire (AMO), il est présenté aux agents de la Caisse Nationale d'Assurance Maladie (CANAM) pour remplir les formalités avant le paiement.

- Si c'est un patient qui bénéficie d'une réduction (personnes âgées, élèves et étudiants, personnel socio sanitaire), il est dirigé vers le chef du bureau des entrées pour se faire enregistrer avant d'aller à la caisse.

- Si c'est un cas social (titulaire d'un certificat d'indigence, et enregistré au niveau de l'Agence Nationale d'Assistance Médicale (ANAM)), il est guidé vers les agents du service social qui assurent la prise en charge.

Pour les hospitalisations, l'usager est alors enregistré dans le registre des admissions et un billet d'hôpital lui est remis. Il va alors au guichet pour s'acquitter de la provision.

A sa sortie, le patient se présente muni de la partie administrative de son billet d'hôpital au bureau des entrées où il récupère sa fiche de sortie et règle le solde. Il se présente ensuite à la caisse pour régler le solde de ses frais de séjour.

\section{6 - Gestion des ordures et des déchets médicaux}

La mise en place du comité technique d'hygiène et de sécurité (CTHS) est en cours. Le processus de désignation des représentants des différentes catégories du personnel devant y siéger a déjà commencé. L'hôpital dispose aussi d'un incinérateur fonctionnel pour l'incinération des déchets biomédicaux. Le nettoyage des locaux de l'Hôpital du Mali est confié à un prestataire privé du nom de Sneig Sarl qui en assure le ramassage au moins deux fois par semaine. Les activités de nettoyage sont effectuées par deux groupes d'agents de Sneig Sarl. Un premier groupe assure le nettoyage le matin avant le démarrage des activités et le second prend le relai le soir après la fin des activités. Durant le déroulement des activités, des manœuvres sont postés dans les services pour effectuer des interventions ponctuelles de nettoyage chaque fois que la situation d'un pavillon l'exige. Des agents de Sneig Sarl sont aussi désignés pour le nettoyage ponctuel de la cour de l'hôpital et pour la salubrité des toilettes extérieures.

\section{7 - Gestion de la sécurisation des locaux de l'Hôpital du Mali}

Le gardiennage des locaux de l'hôpital du Mali est confié à un prestataire privé du nom de global sécurité qui en assure la sécurisation des locaux de jour comme de nuit. Les activités de gardiennage de global sécurité sont effectuées par deux groupes d'agents composés de gardiens et de brancardiers. Ces derniers ont pour rôle d'aider les patients en les amenant dans les endroits appropriés soit dans des brancards, soit dans des fauteuils roulants. 


\section{8 - Gestion des activités sociales de l'Hôpital du Mali}

Au plan social, les activités suivantes ont été menées : l'accueil et l'orientation des malades, l'animation de séances de communication pour le changement de comportement auprès des malades hospitalisés et de leurs accompagnants, participation à la prise en charge alimentaire des malades hospitalisés indigents avec ou sans certificat d'indigence, enquête sociale auprès des indigents, la participation aux évènements sociaux (baptêmes, mariages, décès, etc.) concernant le personnel. L'atmosphère de travail à l'hôpital est bonne dans l'ensemble. A cet effet, le contact entre la direction, les chefs de services et même l'ensemble du personnel est permanent. Les problèmes rencontrés sont discutés à divers niveaux hiérarchiques et des solutions appropriées sont généralement trouvées.

\section{9 - Gestion des activités de buanderie de l'Hôpital du Mali}

Cette activité est assurée par un prestataire privé. Au cours de la période dix mille trois cent quatre-vingt draps ; six mille quatre cent vingt blouses ; deux mille cent dix champs ont été lavés et blanchis.

\subsection{0 - Gestion de l'alimentation / Restauration}

Elle est assurée à travers un contrat par un prestataire qui prépare et qui apporte le repas à l'Hôpital du Mali. La restauration a été régulièrement assurée au personnel de garde et aux malades indigents. Pendant neuf mois d'activités, l'hôpital a quotidiennement assuré la fourniture des trois repas (petit déjeuner, déjeuner et dîner).

\subsection{1 - Gestion des activités de formation de l'Hôpital du Mali}

A l'Hôpital du Mali, pour la période du $1^{\text {er }}$ janvier au 30 septembre 2012 : trois internes ont été formés en pédiatrie ; dix - sept internes sont en formation dont deux en chirurgie thoracique, sept en anesthésie - réanimation et huit en médecine; quarante - neuf DES sont en formation dont huit en chirurgie thoracique, deux en gynécologie, vingt - quatre en anesthésie réanimation et quinze médecine.

\subsection{2 - Gestion des Autres contrats de l'Hôpital du Mali}

L'Hôpital du Mali dispose de contrats pour la maintenance en préparation (gaz, réparations des véhicules, plomberie, électricité froid, suivi des extincteurs, équipements biomédicaux etc.). Le service de la maintenance réalise ces contrats et les suivent. Ainsi, il existe des contrats pour chaque type de prestation effectuée au niveau du service administratif et financier (SAF) une copie. 


\subsection{3 - Gestion des partenariats de l'Hôpital du Mali}

$\mathrm{Au}$ niveau de l'Hôpital du Mali, il existe deux (2) conventions majeures, à savoir : la signature de la convention entre l'hôpital populaire de la province de Zhejiang et l'Hôpital du Mali avec dons de matériel le 30 Septembre 2011 ; la signature de la convention avec l'ONG sante diabète le 15 Octobre 2011. En outre, il existe à l'Hôpital du Mali un CES sous régional en endocrinologie en collaboration avec des partenaires français et qui accueille des auditeurs du Benin, du Burkina Faso, de Guinée Conakry, de Côte d'Ivoire, du Sénégal et du Mali.

\section{3 - Aspects de soutien de l'Hôpital du Mali}

Les activités de soutien réalisées au niveau de l'Hôpital du Mali sont: l'hygiène et l'assainissement hospitalier; la Maintenance (biomédicale, informatique, etc....); les activités de communication hospitalière : SIH ; et la gestion de la pharmacie hospitalière. En outre, à l'Hôpital du Mali, nous avons la présence d'un service d'audit interne et d'un contrôleur de gestion.

\section{1- Maintenance hospitalière}

Le service de maintenance joue un rôle transversal à l'hôpital. Comme son nom l'indique, il s'occupe de la maintenance préventive et curative du matériel, des équipements et des infrastructures. Il intervient dans l'identification des besoins de l'hôpital; établit les cahiers des charges de nouvelles acquisitions, participe à l'élaboration des dossiers d'appel d'offres et aux réceptions. Il assure le suivi technique des équipements et des installations. Il participe à l'élaboration des contrats et le suivi de leurs exécutions. La maintenance des équipements du laboratoire, du matériel informatique, du matériel de sécurité, de la climatisation et de l'imagerie médicale est assurée par des prestataires privés.

\section{2 - Le service de santé publique}

\subsection{1 - Le Système d'Information Hospitalier (SIH)}

Le SIH occupe une place importante dans le processus de gestion et d'amélioration de la qualité de l'information hospitalière. Il veille sur le bon fonctionnement des composantes du cycle de gestion de l'information. A ce titre, il assure : la collecte des données ; le contrôle de la qualité des données ; le traitement des données ; l'analyse et l'interprétation des résultats ; et la diffusion et rétro-information des résultats au niveau local, régional et central. Les attributions assignées au SIH se présentent comme suit : appui aux équipes hospitalières en matière d'encadrement de l'implantation des supports de collecte d'information prévues par le système d'information hospitalier ; assistance aux services de soins pour les aider à produire et 
transmettre les rapports statistiques ; coordination des activités en rapport avec l'évolution du système d'information hospitalier ; dynamisation des actions en faveur de la diffusion et de l'utilisation des informations produites par l'hôpital; production des statistiques hospitalières et constitution de bases de données de statistiques hospitalières ; production des tableaux de bord de performance pour l'évaluation interne et externe de l'hôpital ; contrôle interne de la qualité des informations produites ; contribue à la réalisation d'études statistiques et épidémiologiques en vue de l'analyse et de l'évaluation des activités de l'hôpital ; accompagnement du processus d'information

$\mathrm{SIH}$.

\section{3 - Le service informatique}

La mission du service informatique est d'assurer la gestion et la maintenance du matériel et l'administration du réseau informatique et ondulé.

Les tâches assignées au service informatique sont : veille au bon fonctionnement du parc informatique; supervise le réseau informatique; veille à la maintenance du parc informatique ;

veille à la mise à jour des logiciels utilisés; donne régulièrement un avis technique sur l'état des équipements informatiques; donne un avis technique sur l'acquisition des logiciels; et exécute toutes les taches à lui confiées par le supérieur hiérarchique.

Les tâches assignées à la section administration du réseau et le suivi des logiciels sont : assure le bon fonctionnement du réseau informatique ; administre le réseau informatique ; facilite les activités de télé enseignement ; assure la mise à jour des logiciels utilisés ; initie le personnel à l'utilisation de l'outil informatique ; exécute toutes les taches à lui confiées par le supérieur hiérarchique.

Les tâches assignées à la section maintenance du réseau sont : assure la maintenance préventive et corrective du parc informatique; exécute toutes les taches à lui confiées par le supérieur hiérarchique.

\section{4 - La pharmacie hospitalière}

\subsection{1 - Les modalités d'approvisionnement de la pharmacie}

$\mathrm{Au}$ départ, il a été mis en place un stock initial fourni par la Pharmacie Populaire du Mali (PPM) qui est le principal fournisseur de la pharmacie. Il faut signaler que la pharmacie a reçu près de $40 \%$ de ses besoins de stocks initial exprimés. Les produits non disponibles au niveau de la Pharmacie Populaire du Mali (PPM) qui a un contrat d'approvisionnement avec l'Etat, sont commandés par appel d'offre. Le renouvellement des stocks de médicaments est effectué sur les recettes de la pharmacie. La pharmacie 
bénéficie aussi des dons de médicaments, les médicaments des programmes (programme de lutte contre le paludisme, etc...).

\subsection{2 - La distribution des produits pharmaceutiques}

Il existe deux (2) circuits de distribution au niveau de l'hôpital du Mali, à savoir : la distribution vers les services (cessions); et la distribution aux malades via l'officine hospitalière.

\subsection{3 - Les ressources de la pharmacie}

Les ressources du service de la pharmacie sont: le personnel et les équipements alloués à la pharmacie.

\section{5 - L'audit interne}

L'audit interne est une fonction d'évaluation indépendante qui examine et évalue les services et les activités de l'Hôpital du Mali en vue de lui permettre d'améliorer son efficacité et son efficience. L'audit interne assiste la Direction Générale dans l'exercice effectif de ses responsabilités en effectuant des audits et en fournissant des analyses, des évaluations, des recommandations, des avis et des informations à propos des activités auditées. Il y a un auditeur interne à l'Hôpital du Mali.

\section{6 - Le contrôleur de gestion}

Le contrôleur de gestion constitue un démembrement du contrôle financier délocalisé à l'Hôpital du Mali. Il a en charge la vérification, et l'apposition du visa du contrôle financier sur les documents dont il a vérifié la véracité et l'objectivité. Il est directement rattaché au Directeur Général de l'hôpital.

\section{4 - Plan d'actions du Projet de Gestion et de Soutien (PGS) de l'Hôpital du Mali}

Dans le plan d'actions, il est fait mention par activité : l'objectif général, l'objectif spécifique, l'activité (prise individuellement), la période de réalisation, le résultat attendu et le responsable de suivi de l'activité. Il faut également signaler que les activités du Projet de Gestion et de Soutien constituent les besoins exprimés par les agents de gestion et de soutien en fonction des affectations (ou recrutement suivant les cas), les formations, les équipements et les moyens logistiques.

NB : le Plan d'actions du Projet de Gestion et de Soutien (PGS) étant très volumineux (ne pourra pas contenir dans cet article) nous retiendrons quelques activités choisies par objectif en guise d'illustration. 


\section{Tableau 4 : Plan d'actions du Projet de Gestion et de Soutien (PGS) (quelques activités choisies par objectif en guise d'illustration)}

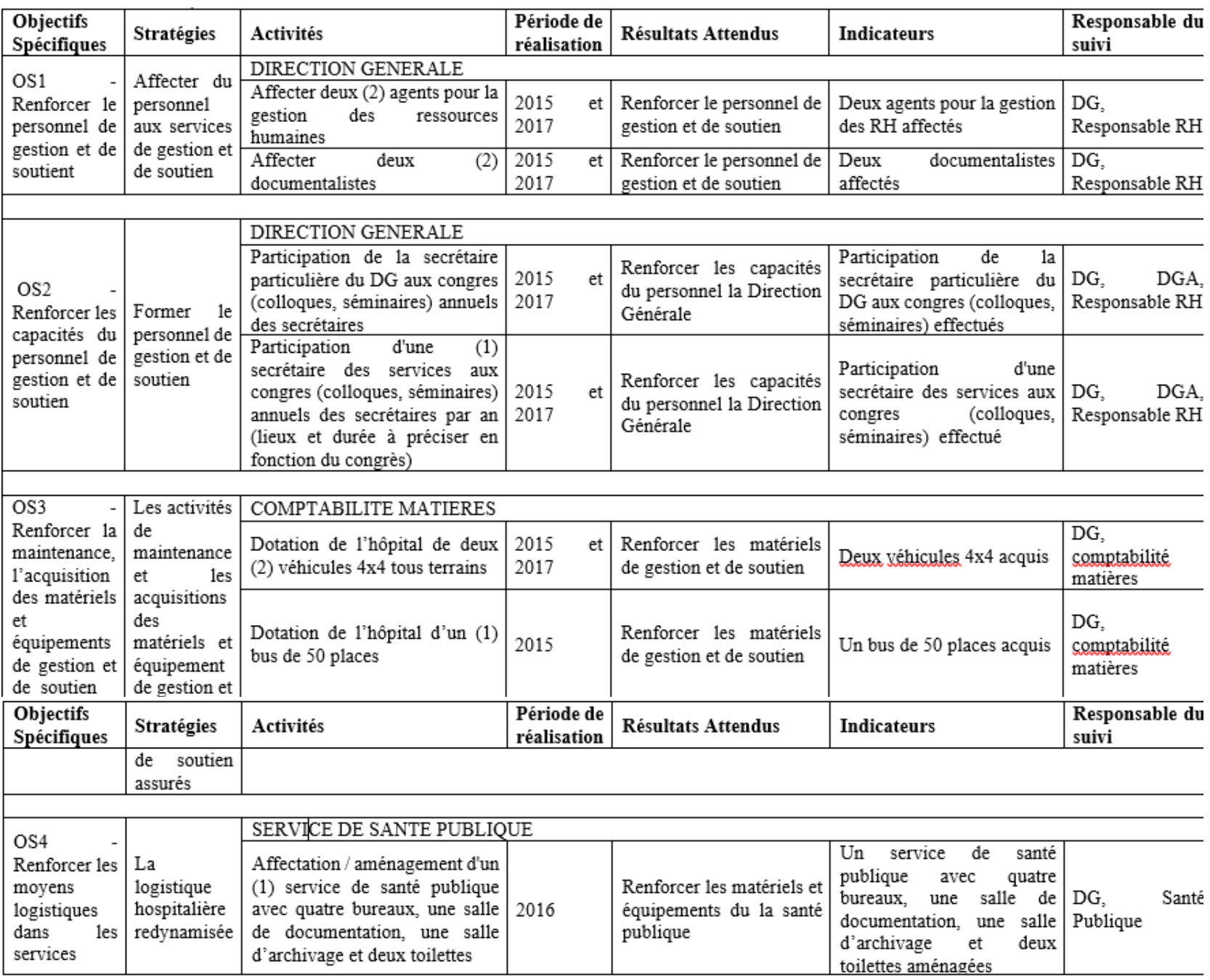

Source : Hôpital du Mali (année 2014), Besoins exprimés par les agents de gestion et de soutien

\section{5 - Impact du Projet de Gestion et de Soutien (PGS) sur l'évolution de l'Hôpital du Mali}

Le rapport d'évaluation conjoint du projet d'établissement 2014 2018 de l'hôpital du Mali réalisé par le service audit interne de l'hôpital du Mali et l'ANEH (Agence Nationale d'Evaluation des Hôpitaux) réalisé en novembre 2019, stipule au chapitre du projet de gestion et de soutien, que le projet d'établissement à permit une organisation de la gestion de l'hôpital du 
Mali. Les deux points qui suivent constituent des acquis pour l'hôpital du Mali ; à savoir :

\section{1 - Valeurs des travailleurs de gestion et de soutien}

Le respect et l'équité sont des valeurs fortes autour desquelles se rassemblent les acteurs de Gestion et de Soutien et constituent aussi la base de l'éthique et de la déontologie professionnelle sur lequel les activités administratives et de gestion sont effectuées à l'Hôpital du Mali. La gestion hospitalière et les activités de soutien hospitalier, de nos jours, demandent une " technicité et une traçabilité " accrues. La mise en place des procédures sécurise la démarche projet.

\section{2 - Management hospitalier}

La conception managériale évolue, elle passe d'une conception pyramidale et segmentée vers une approche transversale intra services et inter services. La responsabilité des acteurs est renforcée, supervisée et évaluée par la Direction Générale et l'Agence Nationale d'Evaluation des Hôpitaux (ANEH) de façon annuelle.

La méthodologie privilégie un Projet de Gestion et de Soutien (PGS) pragmatique et réaliste tout en favorisant une démarche participative et des objectifs stratégiques ciblés par l'hôpital. La coopération incontournable avec les autres acteurs hospitaliers et plus particulièrement la communauté administrative est favorisée pour l'élaboration de ce projet.

\section{6 - Discussion sur le Projet de Gestion et de Soutien (PGS)}

\section{1 - Au niveau national et sous régional}

\section{- Au niveau national}

Dans les structures de santé de même niveau au Mali (en occurrence, les hôpitaux régionaux et les Centres Hospitaliers Universitaires (CHU), les hypothèses de départs sont confirmées, parmi ces Etablissements Publiques Hospitaliers (EPH), nous pouvons cités entre autre : Le projet de gestion et de soutien du projet d'établissement 2012 - 2016 de l'Hôpital Régional de Gao, le projet de gestion et de soutien du projet d'établissement 2013 - 2017 du Centre Hospitalier Universitaire d'Odonto - Stomatologie (CHU-CNOS), le projet de gestion et de soutien du projet d'établissement 2013 - 2017 de l'Hôpital Fousseyni DAOU de Kayes (HFDK), le projet de gestion et de soutien du projet d'établissement 2014 - 2018 de l'Hôpital Nianankoro FOMBA de Ségou (HNFS). Les projets d'accueil, de gestion et de soutien de l'ensemble de ces structures de 2012 à 2018 ont été misent en place. Ils constituent la première vague des projets qui répondent aux exigences édictées dans le guide d'élaboration des projets d'établissement au Mali de l'ANEH. Ces projets ont contribué de façon 
considérable à l'amélioration des aspects de gestion et de soutien dans ces hôpitaux. Par rapport à celui de l'hôpital du Mali, ces projets apportent les mêmes solutions aux différentes structures ci haut citées.

\section{- Au niveau sous régional}

Nous pouvons citer le projet de gestion et de soutien du Projet de du projet d'établissement du Centre Hospitalier Régional Heinrich Lübke de Diourbel au Sénégal (DAFF. M. S., (Septembre 2003)), constitue une référence en la matière. Cette structure réalise des projets d'établissement depuis plus d'une vingtaine d'année. Chaque série de projet apporte une solution adaptée à un problème précis. Par rapport à celui de l'hôpital du Mali, en plus des rubriques du projet de l'hôpital du Mali, cet hôpital prend en compte la gestion du climat social (approche sociologique). Nous pouvons également citer le projet de gestion et de soutien de 1'EPH Ain El Hammam en Algérie (BOUADI. S., (sans date précise)). Ce projet d'établissement a permis à cette région d'Algérie d'améliorer la qualité de la gestion hospitalière. Par rapport à celui de l'hôpital du Mali, la différence réside au niveau des choix méthodologiques; sinon, les résultats sont sensiblement les mêmes.

\section{2 - Au niveau international}

Nous choisirons à ce niveau, cinq projets managériaux et sociaux ayant des contextes socio-économiques différents de ceux du Mali.

\subsection{1 - Le Projet managérial du Projet d'établissement 2017-2021 du CHU de Nîmes}

Au-delà de ce socle de valeurs communes aux différents métiers qui composent la communauté hospitalière du CHU, le projet managérial s'articule autour de trois grands objectifs. Ainsi, la responsabilisation des professionnels est l'un des points forts de ce projet. Elle inclut la mise en œuvre d'un dispositif de délégation de gestion, concerté et travaillé au niveau institutionnel, qui doit permettre le renforcement du «copilotage médicoadministratif» des pôles et une responsabilisation accrue des acteurs. Ce processus apportera des niveaux de décision plus adéquats et opportuns pour fluidifier les circuits administratifs et les arbitrages internes. Le projet managérial met l'accent sur l'écoute et la reconnaissance des professionnels à travers la mise en place d'un baromètre social continu, la contractualisation et le suivi des modalités d'organisation dans chaque unité et la mise en œuvre d'une véritable politique d'intéressement collectif. L'accompagnement des professionnels par la poursuite d'une politique de formation affirmée, basée sur des sessions personnalisées, constitue un prérequis indispensable au maintien des bonnes pratiques et à 
l'accompagnement de l'évolution des prises en charge. L'objectif est d'accompagner l'ensemble des cadres (médicaux et non médicaux) dans leurs actions du quotidien comme dans leurs responsabilités managériales.

\section{Le Projet social du Projet d'établissement 2017-2021 du CHU de Nîmes}

Le projet social répond à des objectifs généraux, répartis en trois thématiques : performance de la gestion des ressources humaines au service des patients et des professionnels, Qualité de vie au travail (QVT) et Responsabilité sociétale et environnementale (RSE). Un parcours professionnel facilité permettra à des professionnels déjà expérimentés d'accéder au CHU, qui favorisera également l'emploi des jeunes et des publics en difficultés pour attirer et fidéliser de nouveaux talents. Il s'agira, dès l'amont du recrutement, de diffuser largement, notamment via une communication de type " marque employeur », les valeurs de l'institution et mettre en exergue le dynamisme et les forces du CHU pour développer son attractivité. L'accueil des nouveaux agents va être repensé de manière à faciliter l'intégration et à développer le sentiment d'appartenance. En ce sens, des actions visent à étendre, à l'ensemble du personnel, le dispositif de tutorat, déjà initié dans les services de soins et à développer l'apprentissage. Des missions de service civique seront à nouveau proposées, après l'expérience mise en place en 2017, pour de jeunes volontaires désireux de s'engager aux côtés d'une institution sanitaire et citoyenne de premier plan. De nouvelles formules des Journées d'accueil des nouveaux arrivants favorisent l'interactivité et les échanges, tout en créant du lien.

\subsection{2 - Le projet managérial du Centre Hospitalier} Universitaire de Reims : Accompagner au mieux les acteurs de la gouvernance des pôles d'activité

Véritable innovation, le projet managérial vise quant à lui à accompagner les acteurs de la gouvernance des pôles d'activité, afin de promouvoir un pilotage optimal des organisations au service de la qualité des prises en charge. Cet accompagnement repose notamment sur des formations adaptées à l'évolution du système de santé et des temps d'échange au bénéfice de la communication managériale. Au-delà de l'optimisation du fonctionnement des pôles dans le cadre d'une politique de gestion prévisionnelle des métiers et des compétences, l'objectif retenu est d'amener à une réflexion d'ensemble sur la bienveillance et l'éthique professionnelle. 


\section{Le projet social du Centre Hospitalier Universitaire de Reims :} Moderniser les relations sociales et assurer le développement des compétences professionnelles

Le projet social prévoit, d'une part, le développement des compétences des professionnels pour leur permettre de s'adapter à un contexte technique évolutif et de s'approprier les nouvelles formes de prise en charge au service des patients. Cet axe repose sur un travail actif de gestion prévisionnelle des métiers et des compétences, en vue de cibler les besoins et de planifier les formations les plus adaptées. D'autre part, il s'attache à permettre une adaptation et un maintien dans l'emploi des professionnels inaptes. Un autre enjeu essentiel concerne le développement de la qualité de vie au travail. Il repose sur la modernisation des relations sociales, en vue de créer un contexte favorable à l'expression des besoins des professionnels et à développer des valeurs sociales partagées, la sécurisation des environnements de travail et des parcours professionnels et le renforcement de la communauté médicale, notamment par des perspectives offertes aux jeunes praticiens.

\subsection{3 - Le projet de gestion du Centre Hospitalier Universitaire de Niort}

Le projet de gestion de l'établissement comporte plusieurs volets structurant pour l'établissement, à savoir : une démarche de développement durable ; la création d'un $11^{\text {ème }}$ pôle ; la politique spécifique «politique achat $\gg$.

\section{Le projet de social du Centre Hospitalier Universitaire de Niort :}

Le projet social a pour objectif de définir les grands axes de la politique de gestion des ressources humaines du Centre Hospitalier de Niort pour les années 2013 à 2017, politique qui vise à assurer la compétence, la motivation, l'adaptation du personnel aux besoins des patients. Il doit permettre de mieux connaître, reconnaître, mobiliser et valoriser les ressources humaines pour accompagner les évolutions prévues par le projet d'établissement.

\subsection{4 - Le projet d'établissement du foyer Bernard Delforge : le foyer}

Bernard Delforge : est un établissement médico-social ; réalise des prestations de services, des projets de prise en charge globale et individualisée ; est une structure se trouvant dans une perspective participative, développementale et d'intégration sociale de la personne handicapée. 


\section{Gestion administrative et financière du foyer Bernard Delforge}

Le service administratif et financier assure la gestion globale de l'établissement tant sur les plans administratifs, financier, que dans le cadre de la gestion des ressources humaines. Les acteurs de ce service travaillent également en synergie et en collaboration avec les instances spécifiques concernées dans le secteur médico-social. Ce service se compose de 5 professionnels sous la responsabilité de la Directrice : la Responsable Administrative et Financière ; l'Assistante de Direction ; le Comptable ; le Comptable Ressources Humaines ; et la Secrétaire d'accueil.

Tous ces professionnels ont des formations spécifiques, ont des tâches et des rôles complémentaires dans chacun des principaux champs d'intervention du service, qui sont : la gestion des personnes hébergées (accueil, facturation, réversion...) ; la gestion des ressources humaines (Embauche, formation, gestion du personnel, pointage, salaires...) ; la gestion comptable (budget et suivi, bilan, trésorerie, commandes, suivi des stocks, inventaires...).

\section{Le projet social du foyer Bernard Delforge}

En articulation avec les autres composantes du projet d'établissement, le projet social repose sur les 4 principes suivants : la qualité et l'amélioration des conditions de vie au travail ; la gestion prévisionnelle des emplois, des métiers et des compétences, le développement d'une politique de communication, de concertation, de négociation : le dialogue social ; la formation continue et la promotion professionnelle; et la démarche qualité et la gestion des risques professionnels.

A travers cette déclinaison de grands axes d'amélioration, les professionnels concernés, ont été représentés par un groupe de travail, dont la réflexion s'est portée sur la qualité des pratiques professionnelles, ainsi que les outils et moyens, mis à disposition pour les rendre plus efficientes.

Les quatre thèmes sont exposés ci-après en précisant pour chacun d'entre eux, le contexte, les valeurs qui ont guidé la définition des objectifs et des actions, des liens avec d'autres projets en cours.

\subsection{5 - Le projet d'établissement 2013 - 2017 du Centre Hospitalier de la Rochefoucauld :}

Projet de Gestion et Management : Ce projet permet d'affirmer en interne les orientations des politiques publiques qui seront mises en œuvre dans le cadre du projet d'établissement.

Les principaux axes stratégiques abordés sont : améliorer les structures d'accueil des patients et résidents ; développer les coopérations sur le territoire en lien avec le GCS (Groupement de Coopération Sanitaire); 
développer le projet de création d'une MSP (Maison de Santé Pluridisciplinaire) adossée à l'hôpital ; créer de nouvelles unités Alzheimer identifiées; dédoubler les chambres à deux lits.

Les principaux axes financiers abordés sont : renforcer le contrôle financier et le développement de la comptabilité analytique et optimiser la gestion de la masse salariale.

Les principaux axes de la logistique abordés sont : améliorer et renforcer la sécurité incendie; développer la politique de développement durable; renforcer les coopérations externes à travers le GCS ; améliorer la performance de nos achats et de notre prestation repas.

Projet Social : Garant de la cohésion et de la dynamique des Ressources Humaines, le projet social est négocié par le Directeur et les organisations syndicales représentatives de l'établissement. Il définit les objectifs généraux de la politique sociale de l'établissement ainsi que les mesures permettant la réalisation de ces objectifs. Ce projet entend donner un signe fort au personnel. Voici les différents axes à développer qui ont été retenus : améliorer l'information et la communication institutionnelle; adopter la Gestion prévisionnelle des métiers et des compétences (GPMC) en Ressources Humaines (afin de valoriser l'agent dans son métier et ses compétences plutôt que dans une pure logique statutaire de grade); développer l'attractivité et la fidélisation des agents ; développer et améliorer la reconnaissance au travail des agents ; faire vivre le document unique ; prévenir et gérer l'absentéisme ; développer la politique de santé publique et de prévention pour le personnel; formaliser l'attention à porter aux agents en période de retour à l'emploi ; valoriser l'implication des agents à travers une politique d'intéressement.

\section{Conclusion}

En conclusion pour ce qui concerne la mise en place du projet de Gestion et de Soutien (PGS) de l'Hôpital du Mali, nous dirons que le projet de gestion et de soutien est maillon essentiel du Projet d'Etablissement (PE). Le Projet de Gestion et de Soutien (PGS) de l'Hôpital du Mali, dans le cadre $\mathrm{du}$ Projet d'Etablissement Hospitalier (PEH) est réalisé par deux commissions, qui sont: le Comité Technique d'Etablissement (CTE) et le Comité Technique d'Hygiène et de Sécurité (CTHS). Le Comité Technique d'Etablissement (CTE) étudie et de donne son avis sur les questions relatives aux conditions de travail dans l'hôpital. Le Comité Technique d'Hygiène et de Sécurité (CTHS) de l'Hôpital du Mali quant à lui étudie et donne son avis sur la protection, l'hygiène, la sécurité des soins et celle des personnes et des biens au sein de l'Hôpital.

Le projet de gestion et de soutien permet (dans son application) à l'hôpital de manager les hommes et de gérer les biens selon les méthodes 
mettant en avant le sens de la responsabilité et l'utilisation efficiente des moyens. Le projet de gestion et de soutien vise à identifier toutes les mesures non médicales qui devront être prises pour permettre à l'hôpital de mener à bien les missions qui lui sont assignées. Elles concernent : d'une part la gestion de l'Hôpital du Mali ; et d'autre part les activités de soutien qui sont réalisées par l'Hôpital du Mali. L'amélioration de la qualité de la gestion et du soutien des activités constitue la préoccupation majeure de la Direction Générale de l'Hôpital du Mali. Le renforcement des capacités des agents de gestion et de soutien a eu une répercussion certaine sur le management global de l'Hôpital du Mali. Le rapport d'évaluation du projet d'établissement de l'hôpital du Mali (Service Audit Interne de l'Hôpital du Mali et ANEH (Agence Nationale d'Evaluation des Hôpitaux), (novembre 2019)) précise que ce projet réalise un taux d'atteinte de $70 \%$ environ. Ainsi, à la lumière de ce qui précède, nous pouvons donc affirmer que les hypothèses de départs ont été confirmés; car le projet de gestion et de soutien a eu des répercussions sur la gestion de l'Hôpital du Mali. Il y a eu une nette amélioration des conditions des agents de gestion et de soutien

\section{References:}

1. Agence Nationale d'Evaluation des Hôpitaux (ANEH), (Octobre 2010), «Guide d'élaboration $d u$ Projet d'établissement des Hôpitaux».

2. BAH. O. (soutenu le 20 juin 2019); "Projet quinquennal 2014 2018 d'établissement hospitalier : cas de l'Hôpital du Mali », Thèse de doctorat, Institut de Pédagogie Universitaire (IPU) de Bamako, Mali.

3. BOUADI. S, (sans date précise), «le projet d'établissement hospitalier : un nouvel instrument au service du management hospitalier. Cas de l'EPH Ain El Hammam ». Mémoire de maitrise, faculté des Sciences Economiques et de Gestion de l'Université Mouloud MAMMERI de Tizi Ouzou, Algérie.

4. Centre Hospitalier Universitaire (CHU) de Nîmes, (2016) ; « Projet d'établissement 2017-2021 du Centre Hospitalier Universitaire (CHU) de Nimes: projet $n^{\circ} 6$ : projet managérial et projet $n^{\circ} 7$ : projet social», site : http://www.chu-nimes.fr/docs/presse/projetetablissement-chu-nimes-2017-2021.pdf.

5. Centre Hospitalier Universitaire (CHU) de Niort, (2017); «Projet d'établissement 2018-2022 du Centre Hospitalier Universitaire (CHU) de Niort, Projet gestion et Projet social». site : https://www.ch-niort.fr/hopital-au-service-du-territoire/le-projetdetablissement. 
6. Centre Hospitalier Universitaire d'Odonto - Stomatologie (CHUCNOS), (2012), "Projet de gestion et de soutien du projet d'établissement 2013 - 2017 du Centre Hospitalier Universitaire d'Odonto - Stomatologie (CHU-CNOS) ».

7. Centre Hospitalier Universitaire (CHU) de Reims, (2015); « Projet d'établissement 2016-2020 du Centre Hospitalier Universitaire (CHU) de Reims, Projet managérial et le Projet social». site du CHU de Reims : https://www.chureims.fr/ckeditor_assets/attachments/103/projet_etablissement_synth ese_2016_-_2020.pdf.

8. Centre Hospitalier de la Rochefoucauld, (2012), «le projet d'établissement 2013 - 2017 du Centre Hospitalier de la Rochefoucauld: Projet de Gestion et Management et Projet social ». site : http://www.ch-larochefoucauld.fr/le-projet-d-tablissement.

9. DAFF. M. S., (Septembre 2003), «La Réforme Hospitalière au Sénégal et l'Accessibilité Financière aux Soins: quelles stratégies pour le Centre Hospitalier Régional Heinrich Lübke de Diourbel?", Mémoire de $3^{\text {ème }}$ cycle de l'ENA de Dakar, Sénégal.

10. Décret $\mathrm{n}^{\circ}$ 10-316/P-RM (03 juin 2010), «fixant l'organisation et les modalités de fonctionnement de l'hôpital du Mali».

11. Foyer Bernard DELFORGE, «Projet d'établissement 2014-2019 du Foyer Bernard DELFORGE, Projet de gestion administrative et financière et Projet social - qualité - gestion $R H$ et $R P »$. site : https://www.foyerbernarddelforge.com/projet-detablissement/gestion-administrative-et-financiere et https://www.foyerbernarddelforge.com/projet-d-etablissement/leprojet-social-qualite-gestion-rh-et-rp.

12. Hôpital Fousseyni DAOU de Kayes (HFDK) (2012), «Projet de gestion et de soutien du projet d'établissement 2013 - 2017 de l'Hôpital Fousseyni DAOU de Kayes (HFDK) ».

13. Hôpital du Mali, deuxième session du Conseil d'Administration (année 2012), « rapport d'activités $2011 》$.

14. Hôpital Nianankoro FOMBA de Ségou (HNFS) (2013), «Projet de gestion et de soutien du projet d'établissement 2014 - 2018 de l'Hôpital Nianankoro FOMBA de Ségou (HNFS) ».

15. Hôpital Régional de Gao, (2011), « Projet de gestion et de soutien du projet d'établissement 2012 - 2016 de l'Hôpital Régional de Gao ».

16. Loi $\mathrm{n}^{\circ}$ 10-010 AN-RM (20 mai 2010), "portant création de l'hôpital du Mali ». 
17. Service Audit Interne de l'Hôpital du Mali et ANEH (Agence Nationale d'Evaluation des Hôpitaux)., (novembre 2019); «Evaluation du projet d'établissement 2014 - 2018 de l'Hôpital du Mali». 
ANNEXE

\section{FORMULAIRE D'ENQUETE - PROJET D'ACCEUIL, DE GESTION ET DE SOUTIEN} DE L'HOPITAL DU MALI

\section{A. Identification de l'enquêté :}

Grille d'évaluation pour le personnel

(Voir la carte professionnelle si possible)

Nom :

Prénom :

Poste occupé :

Service :

\section{B - Administration}

Q1: Gestion administrative de l'hôpital

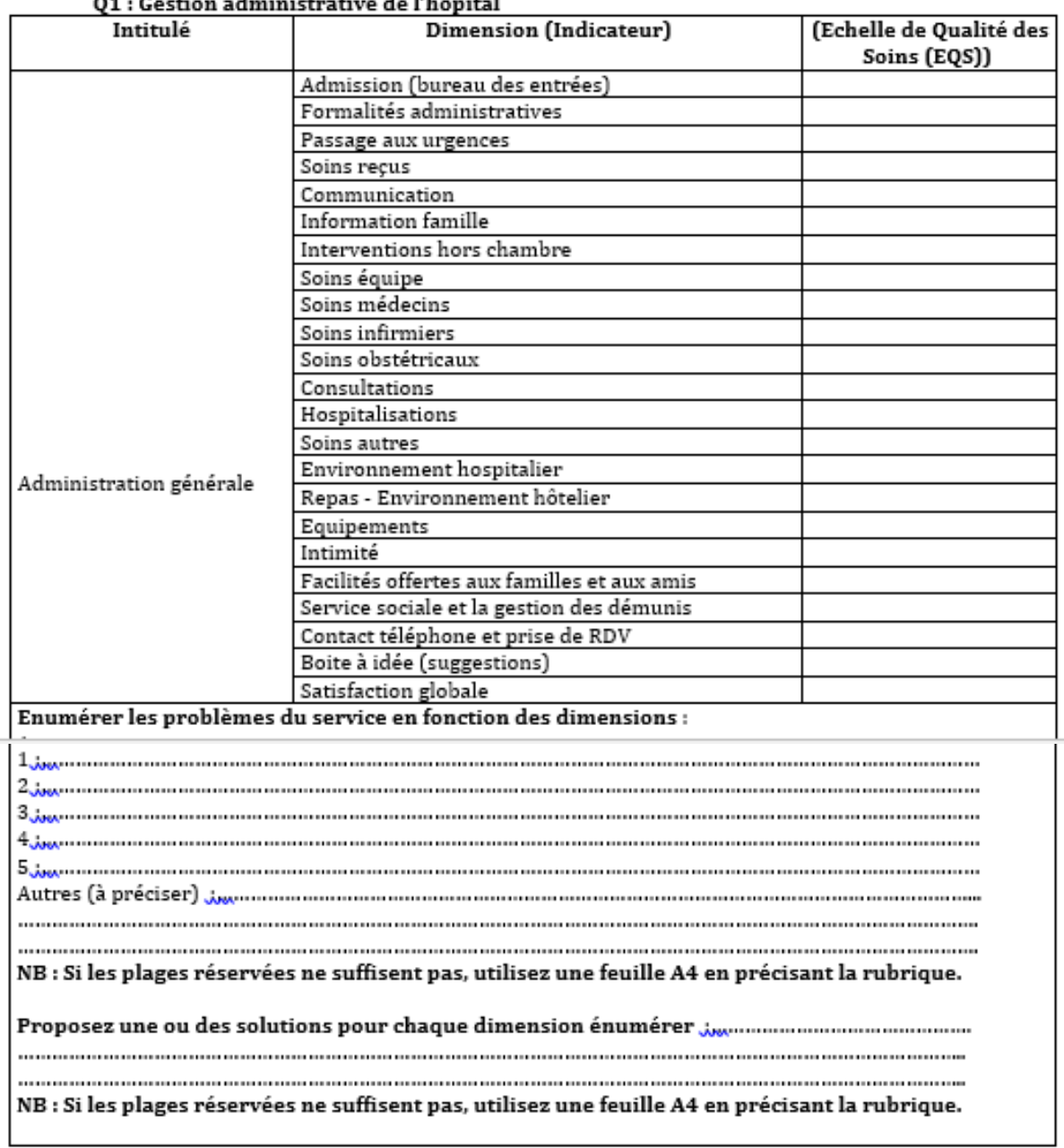

।

Nom et Prénom de L'enguêtteur:
Date : $\ldots . . . / 12 / 2014$

Signature de l'enquêteur: 